\title{
The role of Tre6P and SnRK1 in maize early kernel development and events leading to stress-induced kernel abortion
}

\author{
Samuel W. Bledsoe ${ }^{1}$, Clémence Henry ${ }^{2}$, Cara A. Griffiths ${ }^{3}$, Matthew J. Paul ${ }^{3}$, Regina Feil ${ }^{4}$, John E. Lunn ${ }^{4}$,
} Mark Stitt ${ }^{4}$ and L. Mark Lagrimini ${ }^{5^{*}}$

\begin{abstract}
Background: Drought stress during flowering is a major contributor to yield loss in maize. Genetic and biotechnological improvement in yield sustainability requires an understanding of the mechanisms underpinning yield loss. Sucrose starvation has been proposed as the cause for kernel abortion; however, potential targets for genetic improvement have not been identified. Field and greenhouse drought studies with maize are expensive and it can be difficult to reproduce results; therefore, an in vitro kernel culture method is presented as a proxy for drought stress occurring at the time of flowering in maize (3 days after pollination). This method is used to focus on the effects of drought on kernel metabolism, and the role of trehalose 6-phosphate (Tre6P) and the sucrose non-fermenting-1-related kinase (SnRK1) as potential regulators of this response.
\end{abstract}

Results: A precipitous drop in Tre6P is observed during the first two hours after removing the kernels from the plant, and the resulting changes in transcript abundance are indicative of an activation of SnRK1, and an immediate shift from anabolism to catabolism. Once Tre6P levels are depleted to below $1 \mathrm{nmol} \cdot \mathrm{g}^{-1} \mathrm{FW}$ in the kernel, SnRK1 remained active throughout the $96 \mathrm{~h}$ experiment, regardless of the presence or absence of sucrose in the medium. Recovery on sucrose enriched medium results in the restoration of sucrose synthesis and glycolysis. Biosynthetic processes including the citric acid cycle and protein and starch synthesis are inhibited by excision, and do not recover even after the re-addition of sucrose. It is also observed that excision induces the transcription of the sugar transporters SUT1 and SWEET1, the sucrose hydrolyzing enzymes CELL WALL INVERTASE 2 (INCW2) and SUCROSE SYNTHASE 1 (SUSY1), the clasS II TREHALOSE PHOSPHATE SYNTHASES (TPS), TREHALASE (TRE), and TREHALOSE PHOSPHATE PHOSPHATASE (ZmTPPA.3), previously shown to enhance drought tolerance (Nuccio et al., Nat Biotechnol (October 2014):1-13, 2015).

Conclusions: The impact of kernel excision from the ear triggers a cascade of events starting with the precipitous drop in Tre6P levels. It is proposed that the removal of Tre6P suppression of SnRK1 activity results in transcription of putative SnRK1 target genes, and the metabolic transition from biosynthesis to catabolism. This highlights the importance of Tre6P in the metabolic response to starvation. We also present evidence that sugars can mediate the activation of SnRK1. The precipitous drop in Tre6P corresponds to a large increase in transcription of ZmTPPA.3, indicating that this specific enzyme may be responsible for the de-phosphorylation of Tre6P. The high levels of Tre6P in the immature embryo are likely important for preventing kernel abortion.

Keywords: Maize, kernel culture, drought, starvation, sugar metabolism, trehalose-6-phosphate, SnRK1

\footnotetext{
* Correspondence: mlagrimini2@unl.edu

${ }^{5}$ Department of Agronomy and Horticulture, University of Nebraska-Lincoln,

377I Plant Science, Lincoln, NE 68583-0915, USA

Full list of author information is available at the end of the article
} 


\section{Background}

Drought during flowering impacts most crops, particularly cereals such as maize, and kernel abortion resulting from osmotic stress is one of the main causes of yield loss [1-5]. Under drought, kernel abortion correlates with (i) depleted sucrose and reducing sugar levels, (ii) reduced sucrose degrading enzyme activity and transcript levels, and (iii) increased starch consumption in the kernels. Sink tissues have limited reserves of starch that can help buffer the effects of starvation; however, there is typically an insufficient amount of starch to support the ovary for an extended period of time [6]. Prolonged drought stress changes the embryo's metabolic state, rendering it incapable of using the available sucrose [7], and the damage becomes irreversible through the activation of senescence genes which leads to programmed cell death and kernel abortion [8].

Trehalose ( $\alpha$-D-glucopyranosyl- $(1 \rightarrow 1)$ - $\alpha$-D-glucopyranoside) and its precursor trehalose-6-phosphate (Tre6P) have been found to have many different functions including storage of chemical energy, osmoprotection, pathogen and insect defense and abiotic stress tolerance [9-13]. Trehalose is an important osmotic protectant in bacteria, fungi, and insects where it accumulates to high concentrations [14]. Most plants accumulate only trace amounts of trehalose, where it is unlikely to function as an osmoprotectant [15]. In flowering plants, trehalose is often observed in the pico to nanomolar range, suggesting trehalose and its precursor Tre6P may perform a regulatory or sensing role in plant metabolism with regards to source-sink relations $[16,17]$. Recent evidence points to Tre6P having a central role in sugar sensing [18-23], leading to the proposal that Tre6P is a signal of sucrose availability, and in return regulates sucrose production and utilization [18]. It has also been proposed that Tre6P is a negative feedback regulator of sucrose levels $[24,25]$, perhaps through its interaction with SnRK1 $[20,26,27]$. There are numerous reports where the trehalose pathway has been engineered by constitutively overexpressing TPS or TPP $[28,29]$. In some cases improved drought tolerance was observed, but no mode of action was proposed [30-32]. Perhaps the most conclusive evidence that Tre6P has a role in the plant's response to abiotic stresses was shown using transgenic maize with ectopically produced TPP in the spikelet [33], where it was demonstrated that a reduction in Tre6P levels in the maize ear spikelet improved yield under well-watered conditions, and even more importantly under severe drought stress.

In vitro kernel culture has been selected as a surrogate for drought stress, providing a model system for understanding responses to water deficit and ultimately improving crop yields under drought conditions. Maize in vitro kernel culture was adapted to characterize the impact of sucrose starvation in immature maize kernels (as occurs during a period of water deficit stress) on growth, metabolism, energy status, gene expression and the trehalose pathway. Previous studies using in vitro kernel culture have examined the effects of heat stress [34], hormone synthesis [35, 36], carbon and nitrogen utilization [37], and pollination efficiency [38]. In the experiments presented here, 3 days after pollination (DAP) kernels are placed into axenic culture and subjected to $48 \mathrm{~h}$ of sucrose starvation followed by a $48 \mathrm{~h}$ recovery. Key metabolic indicators of growth and energy status including Tre6P, SnRK1, sugars, sugar phosphates, and organic acids were monitored over the course of the experiment. A detailed characterization of the expression of selected genes having a role in growth, sink strength, sucrose metabolism, sugar transport, starch metabolism provides a picture of the metabolic processes occurring in young embryos during sucrose starvation and recovery.

\section{Results \\ Maize kernel culture}

Zea mays ssp. mays var. B73 was grown in the field with supplemental watering as described in the Materials and Methods. Ears were hand pollinated and harvested 3 DAP. Kernels were removed by dissection, surface sterilized and plated on MS agar. Metabolites and enzymes were analyzed as described in the Materials and Methods. In developing this method, kernels were initially cultured for 15 days to assess long-term growth on sucrose, glucose, fructose and in response to sugar starvation (Additional file 1: Figure S1). Kernels on MS-only medium exhibited no significant growth; although, continued to appear healthy. Kernels cultured on sucrose, glucose or fructose more than doubled in mass over 15 d. There was no significant effect of sugar type or concentration on growth. Kernels were also cultured for 9 days on either MS salts (starvation) or MS salts supplemented with $150 \mathrm{mM}$ sucrose to assess the long term impact of starvation on growth (Additional file 2: Table S1). In a separate experiment sucrose, hexoses, and starch were determined over the course of 11 days in culture, comparing MS-only medium with sucrose-enriched medium (150 mM) (Additional file 3: Figure S2). Levels of all three of these remained constant after an initial change in the first $24 \mathrm{~h}$.

\section{Impact of starvation and recovery on metabolite levels}

The impact of starvation on sugar and starch levels in the kernel was determined. Kernels harvested 3 DAP were placed on either MS-only medium or sucroseenriched medium (150 mM) for $48 \mathrm{~h}$. All kernels were then transferred to sucrose-enriched medium for another $48 \mathrm{~h}$ (recovery). Sucrose levels in kernels subjected to $48 \mathrm{~h}$ starvation were found to rapidly decrease within 
the first $12 \mathrm{~h}$ of culture, whereas sucrose levels in kernels on continuous sucrose increased initially and levelled off within the first $12 \mathrm{~h}$ (Fig. 1). Sucrose levels in starved kernels recovered to levels indistinguishable from kernels receiving continuous sucrose feeding. The data presented are from kernels harvested in 2012. The experiment was repeated in 2013 with no significant difference in the outcome (Fig. 1). In 2013, the calculated rates of kernel sucrose depletion and recovery were similar, $3.19 \mu \mathrm{mol} \cdot \mathrm{g}^{-1} \mathrm{~h}^{-1}$ and $3.55 \mu \mathrm{mol} \cdot \mathrm{g}^{-1} \mathrm{~h}^{-1}$ respectively. Although plants had supplemental irrigation available, the environmental conditions varied greatly between the years (Additional file 4: Table S2). The 2012 growing season was considered a drought year with the highest temperatures and growing degree days (GDD), while the 2013 growing season was more typical for the location.

\section{Impact of starvation on tre6p and other metabolites}

In order to assess the effect of starvation and recovery treatment on maize kernel metabolism, an analysis of metabolites utilized in sucrose and starch synthesis, glycolysis, and the citric acid cycle was performed (Fig. 2; Additional file 5: Figure S3). Sucrose deprivation for $48 \mathrm{~h}$ reduced Glc and Fru to very low levels $\left(<10 \mu \mathrm{mol} \cdot \mathrm{g}^{-1} \mathrm{FW}\right)$, and this decrease was largely reversed by the addition of sucrose in the medium. The glycolytic intermediates Glc6P, Fru6P (intermediates in glycolysis, Suc synthesis and starch synthesis), fructose 1,6-bisphosphate (FBP; an intermediate in glycolysis), and glycerol 3-phosphate (Gly3P; an intermediate in the synthesis of glycerolipids) rose in the sucrose control but remained level or rose only slightly in the starvation treatment, while 3PGA and PEP rose more in the starvation treatment than in the sucrose control. Many of these differences were statistically significant at $48 \mathrm{~h}$ starvation. Pyruvate declined strongly after $12 \mathrm{~h}$ (from 150 to $60 \mathrm{nmol} \cdot \mathrm{g}^{-1} \mathrm{FW}$ ), with a more marked decline in the starvation treatment than the control. Many intermediates in the citric acid cycle remained high in the sucrose control but declined in the starvation treatment, including citrate, aconitate, isocitrate, 2-oxoglutarate, succinate, and malate (Fig. 3). "During the recovery from starvation, the levels of sugar phosphates in starved kernels returned to that of those maintained on continuous sucrose. 3PGA and PEP remained slightly but not significantly higher than in the sucrose control. It was striking that many organic acids did not return to initial levels after recovery on sucrose-containing medium, indicating an inhibition of pyruvate kinase and/or PEP carboxylase.

Sucrose and starch metabolism were also impacted by starvation in the detached kernels. Sucrose deprivation triggered a rapid decrease in S6P followed by a return to control levels after returning to sucrose-enriched medium, while steadily increasing in the continuouslyfed kernels. The decrease in S6P during the first $8 \mathrm{~h}$ of starvation was more marked than for Glc6P and Fru6P which are important in both sucrose and starch

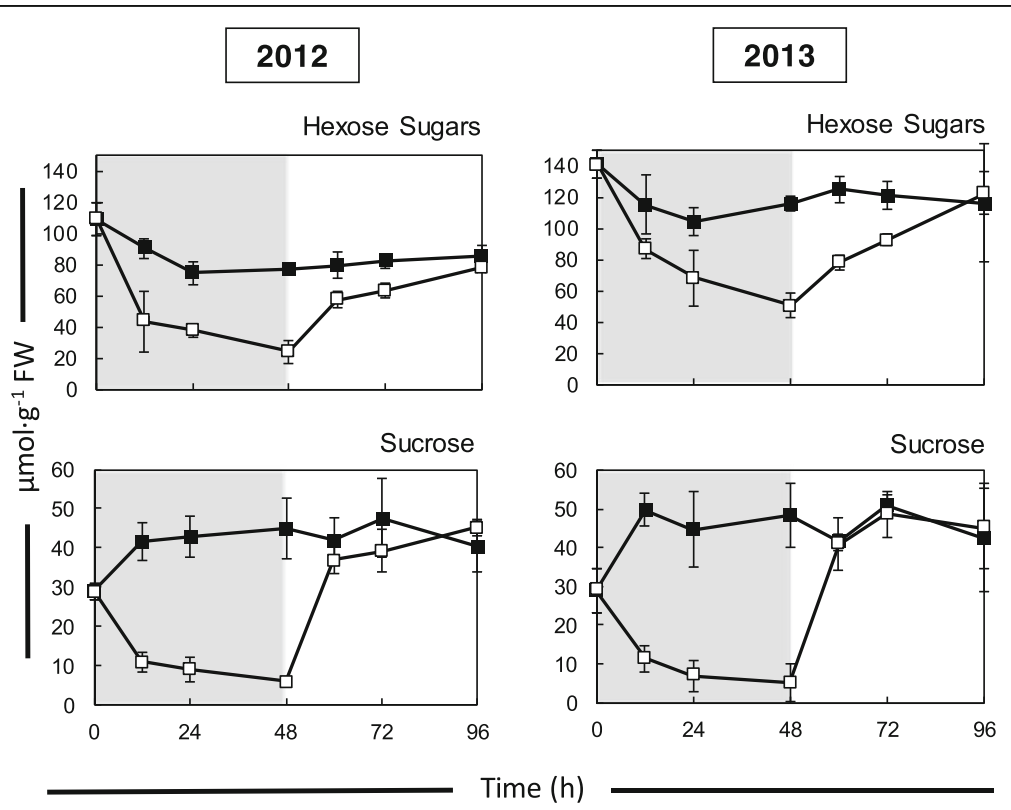

Fig. 1 Comparison of sugar levels for kernels isolated from field grown maize (B73) from the 2012 and 2103 growing seasons. Control kernels on continuous sucrose feeding are indicated with closed (black) squares, and sucrose-starved kernels are indicated with open (white) squares. The shaded area of the graph indicates the starvation period, and the unshaded region represents the recovery stage when starved kernels were allowed to recover on sucrose-enriched medium. The initial time point $(T=0)$ is from after kernel sterilization 


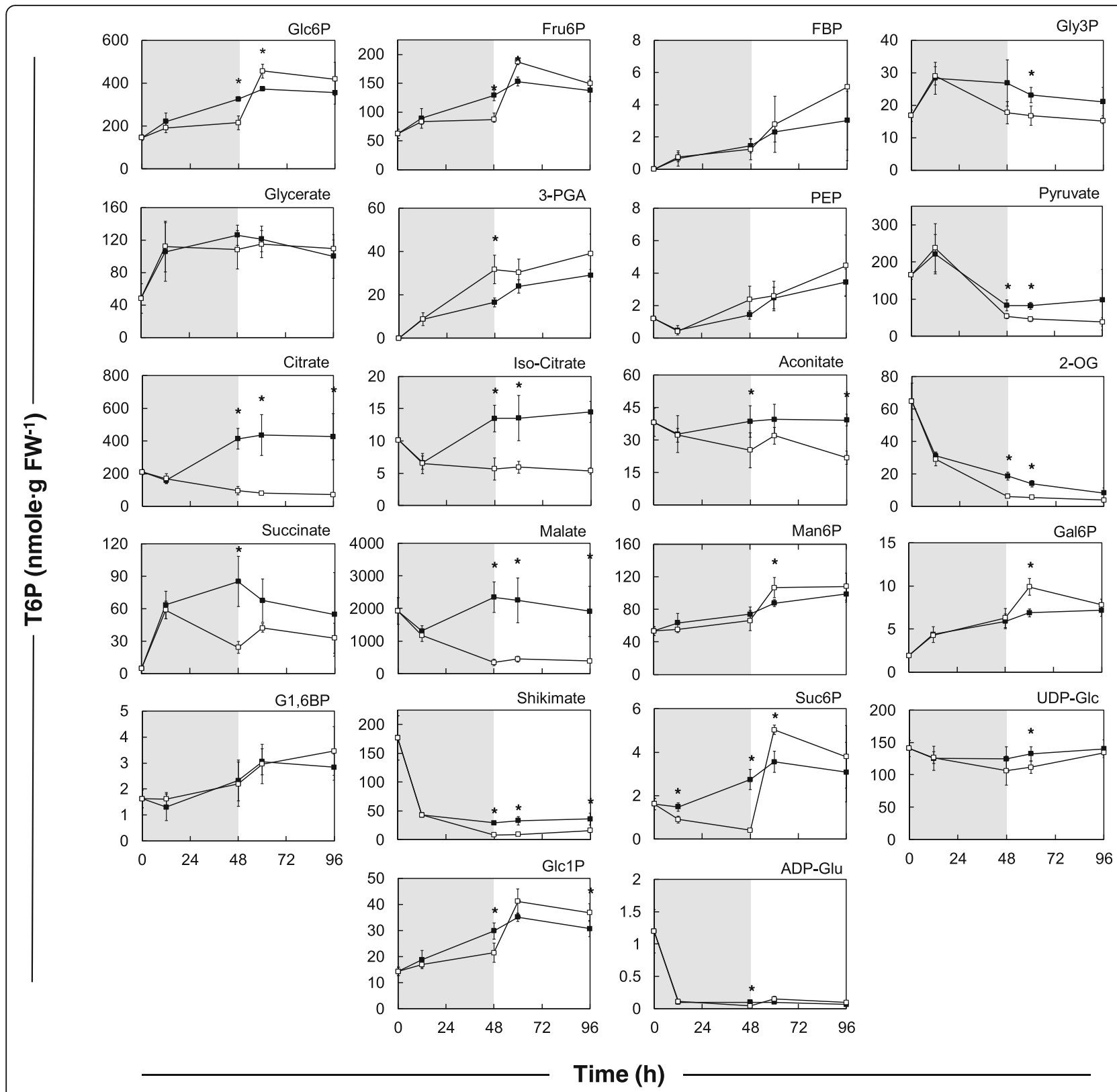

Fig. 2 Effects of sucrose starvation on metabolite levels (on a fresh weight basis) on 3 DAP B73 kernels (2012 season). All metabolites were measured using anion exchange chromatography in tandem with mass spectrometry as described by Lunn et al. [18]. Control kernels on continuous sucrose are indicated with closed squares. Kernels starved for $48 \mathrm{~h}$ (shaded area), then allowed to recover for $48 \mathrm{H}$ on $150 \mathrm{mM}$ sucrose are indicated with open squares. Means are calculated using 4 biological replicates, consisting of 3 plants each. Significance as indicated by asterisks was determined by the Student's t test $(P<0.05)$

synthesis (Fig. 3). There was a strong decrease in ADPGlc and shikimate in the sucrose control as well as the starvation treatment, indicating decreased aromatic amino acid synthesis, although the residual level of shikimate was slightly higher in the sucrose control. The levels of ADPGlc and shikimate remained low after the re-addition of sucrose.

An exceptionally large decrease in Tre6P levels from 54 to $1 \mathrm{nmol} \cdot \mathrm{FW}^{-1}$ was observed in both sucrose fed and sucrose deprived samples within the first $12 \mathrm{~h}$ of culture and maintained throughout the duration of the experiment (Fig. 4). "Tre6P has been shown to stimulate flux through the citric acid cycle via the activation of PEP carboxylase, thus the low levels of Tre6P in excised kernels and the lack of a recovery after re-supplying sucrose could possibly prevent the activation of this enzyme and explain the decrease in organic acids in excised kernels and the inability of sucrose to revert this 


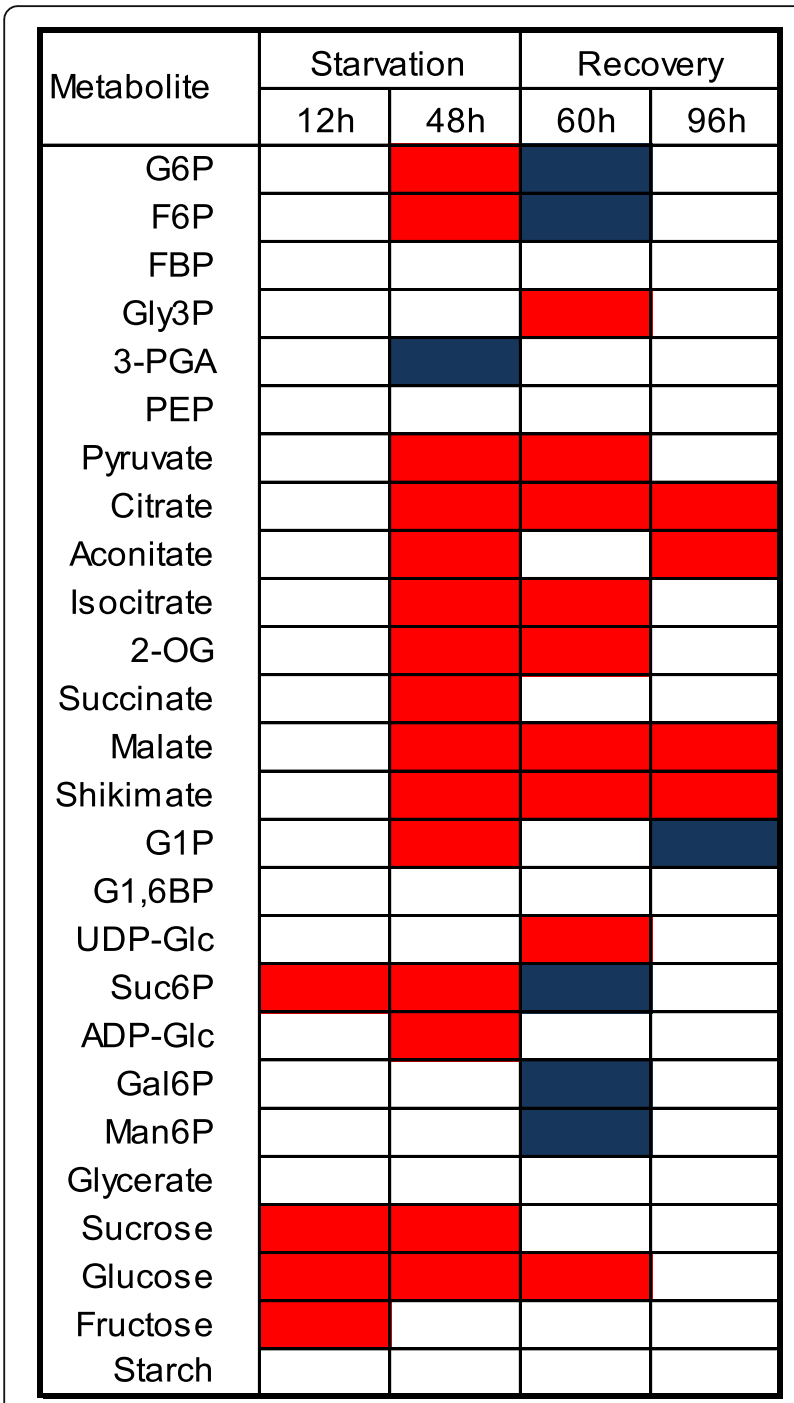

Fig. 3 Heat map indicating the overall effect of sucrose starvation and subsequent recovery on metabolite levels for cultured maize kernels (3 DAP). ANOVA was performed on four independent biological replicates using Microsoft Excel. Significant differences, $P<0.05$, indicated by shaded boxes. Blue indicates treatment had a positive effect over control, and red indicates treatment had a negative effect over control samples. Non-significant differences are indicated with white bars

decrease [25]. The zero time point was not included in Fig. $4 \mathrm{~b}$ so that the smaller later changes in Tre6P, following the large initial decrease, could be seen more clearly. It is now possible to see that sucrose deprivation results in a small further reduction of Tre6P in kernels throughout the duration of the experiment.

Impact of starvation and recovery on in vitro snrk1 activity In order to assess the impact of the starvation and recovery treatments on the stress and sucrose starvation sensor SnRK1 in cultured maize kernels, in vitro SnRK1 activity was determined. It was previously shown that SnRK1

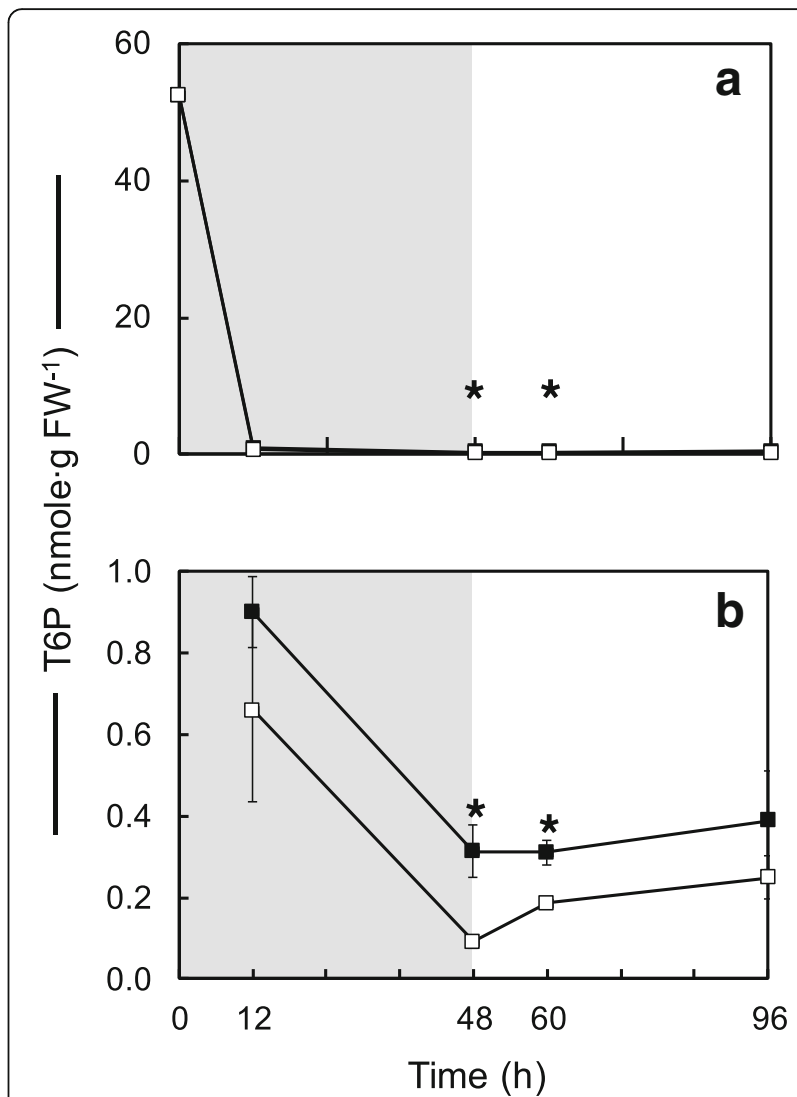

Fig. 4 Effects of sucrose starvation on Tre6P levels in cultured maize kernels (2012 season). Tre6P content was determined for kernel tissue using anion exchange chromatography in tandem with mass spectrometry [18]. a Control kernels on continuous sucrose are indicated with closed squares. Kernels were starved for $48 \mathrm{~h}$ (shaded area), then allowed to recover for $48 \mathrm{~h}$ on $150 \mathrm{mM}$ sucrose are indicated with open squares. $\mathbf{b}$ Region from 12-96 $\mathrm{h}$ is expanded for detail. Means are calculated using 4 biological replicates, consisting of 3 plants each. Significance as indicated by asterisks was determined by the Student's t test $(P<0.05)$. FW, fresh weight

activity in maize kernel extracts was highly sensitive to inhibition by Tre6P [39]. Total and Tre6P-inhibitable (1 mM) SnRK1 activity was determined and graphically presented as Tre6P-inhibitable activity (Fig. 5). Control and starved kernels exhibited a drop in T6P-inhibitable SnRK1 activity after placed in culture which then returned to near initial levels by $96 \mathrm{~h}$ for both treatments. While this occurred monotonically for the continuously-fed control, Tre6P-inhibitable SnRK1 activity rose significantly between 12 and $48 \mathrm{~h}$ in the starvation treatment, and remained elevated $12 \mathrm{~h}$ after sucrose re-addition. Sucrosestarved kernels had significantly higher SnRK1 activity at 48 and $60 \mathrm{~h}$ when compared to control samples.

\section{Impact of starvation and recovery on gene expression}

The effect of sucrose deprivation and recovery on gene expression was examined. Relative gene expression was 

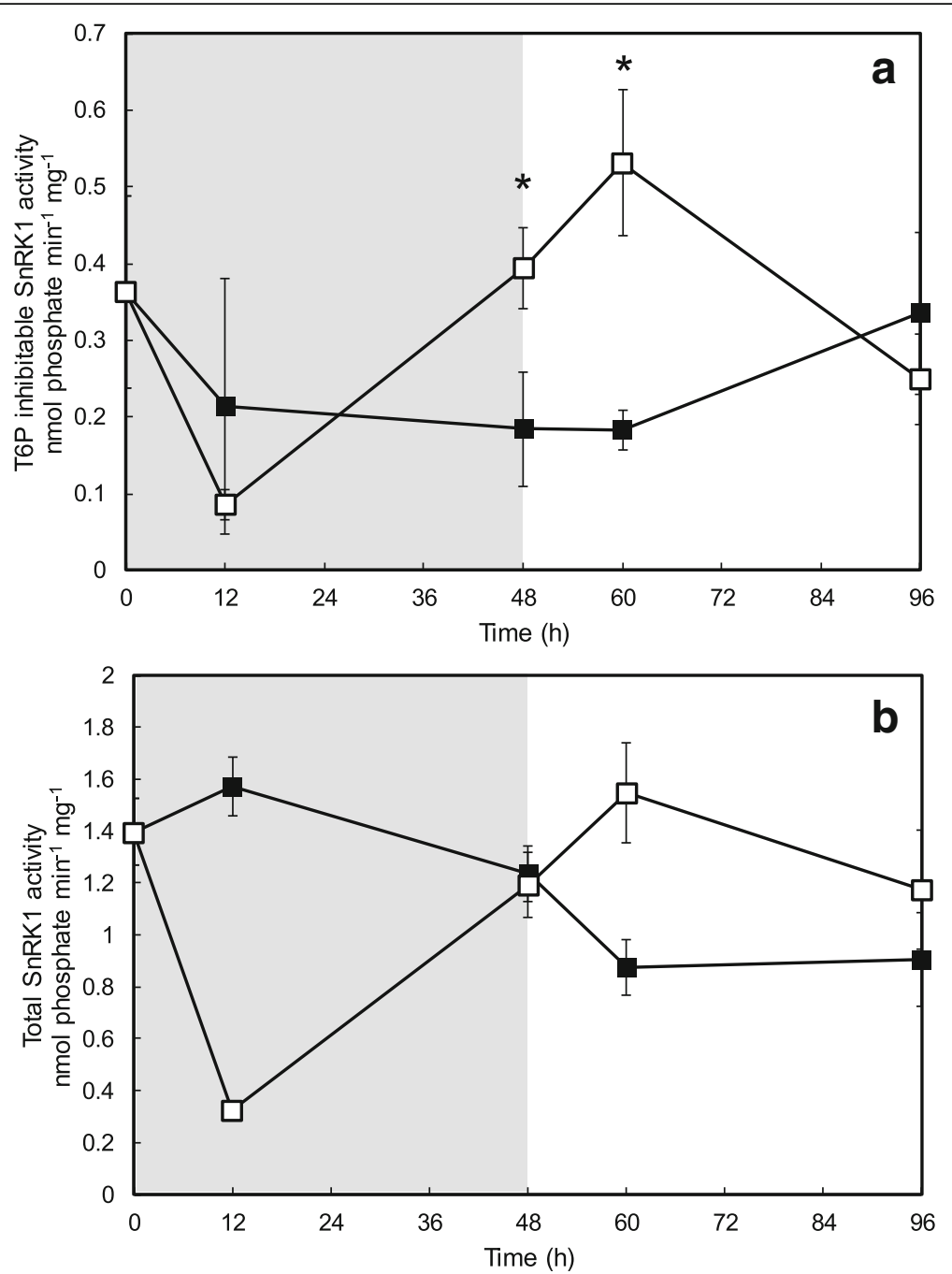

Fig. 5 Tre6P inhibitable in vitro SnRK1 activity for control and sucrose-starved kernels (2012 season). SnRK1 activity was determined in tissue extracts as described by [20]. Exogenous Tre6P $(1 \mathrm{mM})$ was added to determine the fraction of SnRK1 activity sensitive to Tre6P inhibition. Shaded region of graph indicates starvation period for treated kernels, and unshaded region indicates recovery period. Control samples are indicated with filled squares (black) and starved kernels are indicated with open squares (white). Asterisks indicate significant difference from controls $(P<0.05$;

$n=3)$. Standard error is shown with vertical bars

determined by qRT-PCR for a select set of genes involved in the trehalose pathway (TPS, TPP and TRE), SnRK1 target genes and genes involved in sugar metabolism and transport (Fig. 6). Among the TPS genes, the ones encoding class I and Class II enzymes responded differently to sucrose deprivation. The class I gene (ZmTPS1.1), encoding the TPS enzyme with catalytic activity, declined gradually from $12 \mathrm{~h}$ onwards and did not show any significant differences in expression between continuously-fed and sucrose starved kernels. All of the class II TPS genes investigated were induced within the first $8 \mathrm{~h}$ of sucrose deprivation. There was a trend for all class II TPS mRNAs to rise to higher levels in the sucrose deprived samples, but only three (ZmTPSII.2.1, ZmTPSII.3.3 and ZmTPSII.4.1) were found to have significant differences between the control and sucrose starved treatments at either the $12 \mathrm{~h}$ or $48 \mathrm{~h}$ time points (Student's T-test, $\alpha=0.05$ ). This effect was reversed upon the re-addition of sucrose.

In contrast to the class II TPS genes, the $P P$ genes did not respond in such a coordinated fashion. For ZmTPPA.3, there was a large induction of transcript levels in the first $8 \mathrm{~h}$ of culture. For this gene, there was no difference between control and starvation treatment within the first $48 \mathrm{~h}$ of starvation, but gene expression was higher during recovery for the previously starved kernels compared to controls. ZmTPPB.1.3 gene expression was similar to that of ZmTPPA.3, showing a sizable induction in the first $8 \mathrm{~h}$ of culture (Fig. 6). Transcript levels for sucrose-deprived kernels responded to a 


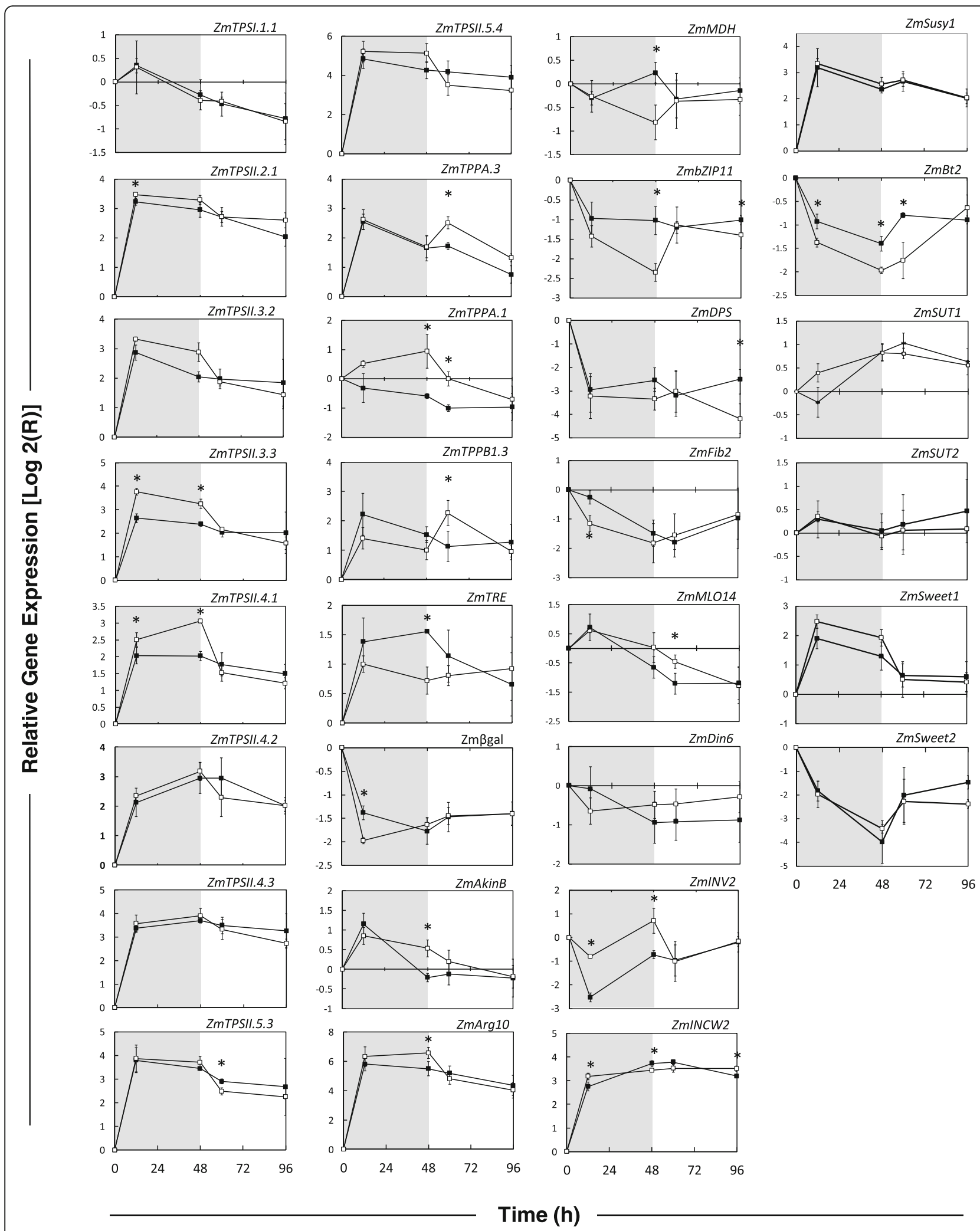

Fig. 6 (See legend on next page.) 
(See figure on previous page.)

Fig. 6 Transcript levels in in vitro grown B73 maize kernels subjected to sucrose starvation and recovery (2012 season). RT-gPCR was performed on total RNA extracted from kernel tissue using oligonucleotide primers specific for various genes involved in sugar metabolism, sensing and transport. White squares indicate kernels subjected to $48 \mathrm{~h}$ of sucrose starvation followed by recovery for $48 \mathrm{~h}$ on $150 \mathrm{mM}$ sucrose. Black squares indicate kernels grown on continuous sucrose. The shaded area on the graph represent the period of sucrose starvation. The white are shows the recovery period on $150 \mathrm{mM}$ sucrose. Significance as reported by the Student's $T$-test is at a $=0.05$ for 3 biological replicates, and indicated by asterisks. Standard error is shown with vertical bars

greater extent to recovery on sucrose. ZmTPPA.1 transcript level was not affected by culture in the presence of sucrose, but displayed a small increase as a consequence of starvation. ZmTRE (TREHALASE) showed an induction in the first $8 \mathrm{~h}$ of culture and was significantly repressed after $48 \mathrm{~h}$ of starvation. ZmTRE mRNA levels returned to that of control samples during recovery on sucrose.

The impact of the starvation and recovery treatment on the SnRK1 signalling pathway was assessed using the transcript levels of nine putative SnRK1 target genes [27]. Primers were designed for maize based on homologous sequences with Arabidopsis: 5 SnRK1 induced genes (ZmßGal, ZmAkinß, ZmArg10, ZmMOL14 and $Z m D I N 6)$ and 4 SnRK1 repressed genes $(Z m M D H$, ZmbZIP11, ZmDPS, and ZmFiB2). Selected SnRK1 target genes responded to both cultivation and sucrose deprivation stress. For stress specific SnRK1 target genes, $Z m A k i n \beta$ has been shown to be induced and $Z m M D H, Z m b Z I P 11$ and ZmFiB2 are repressed [27]. Among the putatively upregulated SnRK1 targets, four responded accordingly: ZmAkin $\beta, Z m A r g 10, Z m D I N 6$ and $Z m M o l 14$ when kernels were placed into culture. Among the putatively downregulated SnRK1 targets, five, ZmßGal, ZmßZIP11, ZmDPS, ZmFib2, ZmMDH, were repressed in response to culturing. However, only $Z m A k i n \beta, Z m A r g 10, Z m M D H$, and ZmßZIP11 transcripts were significantly different in starved kernels when compared to controls at $48 \mathrm{~h}$ into treatment. Putative SnRK1 target gene expression responds to the addition of sucrose in the medium after $48 \mathrm{~h}$ of starvation in a Tre6P independent manner. This can be seen with the transcripts for $Z m A k i n \beta, Z m A r g 10, Z m M D H$, and most noticeably $Z \mathrm{~m} \beta Z I P 11$. "This Tre6P independent response to sugars is also seen with all three TPP genes (ZmTPPA.3, ZmTPPA.1 and ZmTPPB.1.3). After sucrose recovery (48-96 h), there was no significant difference between control and starvation treatment with the exceptions of ZmDPS at $96 \mathrm{~h}$ and ZmMol14 at $60 \mathrm{~h}$. SnRK1 target gene expression data provide further evidence that the SnRK1 pathway is responding to in vitro kernel starvation.

Genes critical to sucrose metabolism; vacuolar invertase (ZmIVR2), cell-wall invertase (ZmINCW2), sucrose synthase (ZmSuSy1), AGPase domain gene BRITTLE2 (ZmBT2), and sucrose transporters (ZmSUT1, ZmSUT2,
ZmSWEET1, and ZmSWEET2) were also assessed for their expression. ZmIVR2 gene expression decreased when kernels were placed into culture, similar to the putatively downregulated SnRK1 target genes. Transcript levels; however, remained significantly higher in starved kernels than controls during the first $48 \mathrm{~h}$ (Fig. 6). Both $\mathrm{ZmINCW} 2$ and $\mathrm{ZmSuSy} 1$ transcripts were induced in the first $8 \mathrm{~h}$ of culture for both control and treated kernels, reflecting the same pattern seen for class II TPS genes and putatively upregulated SnRK1 target genes. $Z m B T 2$ transcript decreased in the first $8 \mathrm{~h}$ of culture and stayed lower in sucrose-starved kernels throughout the experiment. The sucrose transporters ZmSUT1 and ZmSWEET1 showed higher mRNA levels for starved kernels with values approaching significance (Fig. 6). Neither ZmSUT2 nor ZmSWEET2 differed in expression between the control and starved kernels. The expression of three sugar transporters (ZmSUT1, ZmSUT2, ZmSWEET1, and ZmSWEET2) was followed in response to starvation stress in the immature maize kernel. The transcription of ZmSWEET1 and 2 sugar transporters is impacted by the starvation during harvest; however, unlike the other SnRK1-regulated genes, transcription returns quickly to initial levels within 96 h. ZmSWEET1 was strongly induced when kernels were placed into culture, yet mRNA quickly returned to initial levels upon transfer to sucrose-enriched medium. $Z m S W E E T 2$ gene expression showed the opposite response, decreasing during starvation and increasing during recovery. The senescence indicator gene ZmDIN6 [27] did not differ significantly between control and sucrose starved kernels.

The process of harvesting and sterilizing 3 DAP maize kernels imposes an unavoidable degree of stress. It takes $2 \mathrm{~h}$ from the point at which kernels were removed from the ear to plating on nutrient agar. During this $2 \mathrm{~h}$ window all kernels including controls were effectively starved for nutrients. It was observed that excision and sterilization impacts mRNA levels for numerous genes involved in sugar sensing, transport and metabolism. This "excision effect" is independent of the presence of sucrose in the medium. To better understand the excision effect, a separate experiment was conducted to look at gene expression in the initial hours postexcision (Fig. 7). To determine how quickly a change in transcript levels occurred after kernels were removed 


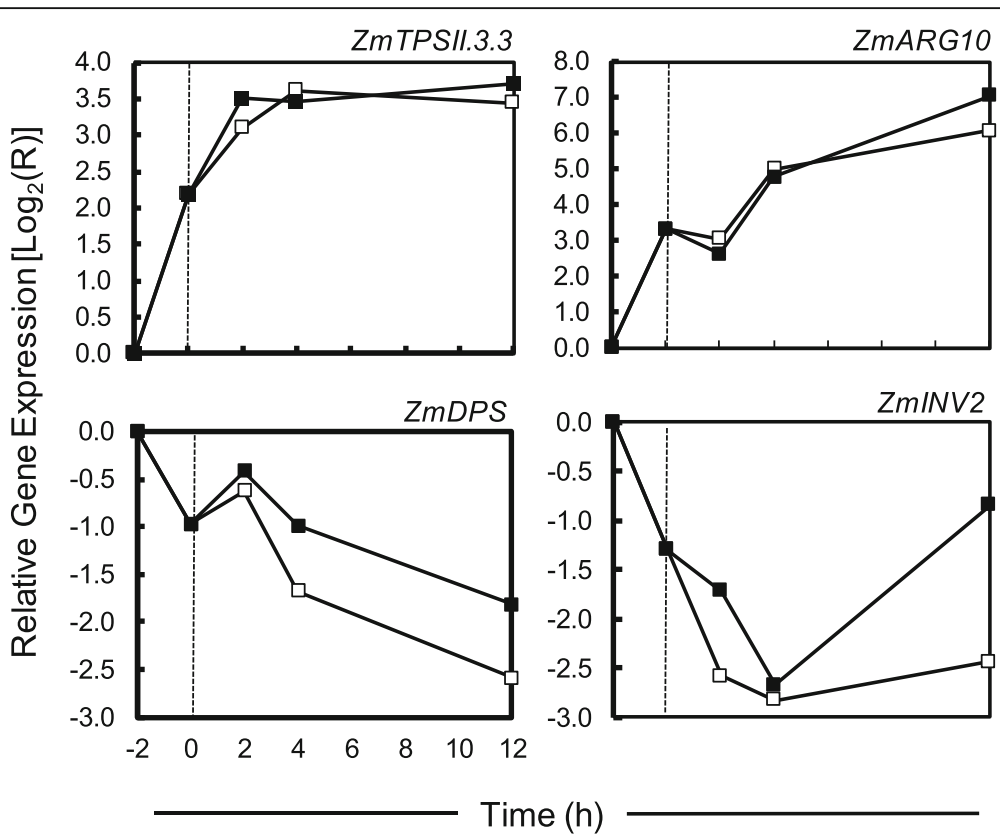

Fig. 7 RT-qPCR was performed on total RNA extracted from maize kernels cultured in vitro on MS complete agar \pm 150 mM sucrose. Open squares indicate kernels starved for sucrose, and closed squares indicate kernels subjected to sucrose starvation. Means are plotted based on 3 biological replicates. At $-2 \mathrm{~h}$ the ears were removed from the plant. The vertical dashed line indicates when kernels were placed on medium

from the ear, we sampled at $-2,0,2,4$ and $12 \mathrm{~h}$. The $-2 \mathrm{~h}$ time point reflects the moment the ears were removed from the plant, and the $0 \mathrm{~h}$ time point is when kernels are placed on nutrient medium. Quantitative RT-PCR was performed for two genes shown to be induced by excision (ZmTPSII.3.3 and ZmARG10) and two excision repressed genes (ZmDPS and ZmIVR2). Transcript levels for these genes were found to change by as much as 8-fold in the two hours it took to excise, sterilize and plate the kernels (Fig. 7). Messenger RNA levels continued to increase or decrease for another $4 \mathrm{~h}$ before levelling off. The 3 DAP kernels were divided into three periods. Duke and Doehlert [40] looked at mRNA levels in 5 DAP maize kernels for $\alpha$-Zein, ß-Zein, Opaque-2, Aldolase, Shrunken-1, Waxy, Shrunken-2, and Brittle-2 at 0, 5, 10, and $15 \mathrm{~d}$ into culture. Transcription increased for each of these genes from 0 to 5 days, then returned to initial levels $\left(25^{\circ} \mathrm{C}\right)$. Although not discussed in their paper, this initial increase in transcription could be the consequence of inflicted starvation during the harvest, as observed in this paper. It is important that interpretations of the data take into consideration possible wounding or excision effects.

\section{Discussion}

\section{Effect of starvation on sugar metabolism and transport}

Sugar uptake is essential to sustain kernel development and leads to kernel abortion if disrupted in any way, i.e. when drought is applied around the time of pollination [41]. In the work presented here, we chose to use an in vitro kernel culture system (in presence or absence of sugar) as a way to mimic disruptions in sucrose supply to the kernel. We then tested how it impacted sugar and starch metabolism and sugar sensing. Sucrose levels increased and hexose levels decreased by only two thirds in the sucrose-fed control kernels. This demonstrates that in our experimental system the sucrose supplied in the media was well taken up by the kernel. It also indicates that there is a limit to how much of the sucrose and hexose can be metabolically processed, and that this metabolic processing is in equilibrium with sucrose hydrolysis. This contrasts with starved kernels in which both sucrose and hexoses decreased dramatically indicating that they are being consumed by catabolic pathways to maintain energy status. This observed equilibrium between metabolic processes and invertase activity has been shown to be maintained by hormones and hexose signals in a feedback regulation loop [42].

We observed no difference in starch levels between the control and sucrose-starved kernels over the course of the $96 \mathrm{~h}$ experiment. One possible explanation is that starch synthesis is irreversibly inhibited in response to the initial $2 \mathrm{~h}$ starvation period. The rapid decline in the expression of the AGPase gene ( $\mathrm{ZmBt} 2)$ as well as lower levels of the starch synthesis intermediate ADP-Glc in both the control and starved kernels supports this explanation. An alternative explanation is that embryos in early development are not in the grain filling stage, where most of the starch is synthesised [43]. 
Interestingly, elevated 3-PGA levels were observed. The glycolytic intermediate 3-PGA, is known to allosterically activate AGPase (regulatory step in starch synthesis) and is generally correlated with high sucrose availability resulting in starch biosynthesis [44-46]. However, in this study the opposite is observed. Lack of starch accumulation, reduced sucrose levels, repressed ZmBt2 mRNA, and low starch biosynthesis metabolites, in particular the dedicated intermediate, ADPGlc, all indicate that starch biosynthesis is being inhibited. Although 3-PGA is high in sucrose-deprived kernels and normally would allosterically activate AGPase, AGPase is also redox-activated as it adjusts to the supply of available carbon [47], sucrose starvation is most likely the reason why the $Z m B T 2$ gene is turned off to a greater extent in the sucrose-deficient kernels even in the presence of elevated 3-PGA.

Whole plant studies have shown that drought stress impacts the metabolism of sucrose, and is negatively correlated with IVR2 and INCW2 enzymatic activity and mRNA transcript levels [31, 48, 49]. These enzymes, as well as Susy1, are responsible for sucrose cleavage into hexose in order for them to be uptaken by the kernels. In our experiments, we observed that $48 \mathrm{~h}$ of starvation induced a strong accumulation of ZmIVR2 transcripts, a small decline of ZmINCW2 transcripts, and did not affect $Z m S u s y 1$ transcripts. This indicates that similarly to ZmSWEET1 and ZmSUT1, ZmIVR2 might be regulated by sucrose availability and induced upon starvation in an attempt to increase the uptake of sugar by the kernel. It also indicates that ZmIVR2 might have a more important function than ZmINCW2 and ZmSusy1 in that response. This hypothesis correlates with previous findings showing that sucrose synthase (Susy) is more important in sucrose catabolism during later development, when most starch synthesis occurs, than in early development $[42,43,50]$.

The SWEET transporters have been recently discovered as a new class of sugar transporters enabling passive diffusion of either sucrose or hexose through membranes according to the concentration gradient of sugars. They have therefore been postulated to be the missing transporters enabling apoplastic sucrose phloem unloading and sugar uptake by sink tissues through passive diffusion [49]. However, little is known about their specific function and localization in different plant tissues and across species [51, 52]. In contrast to the SWEET transporters, the sucrose- $\mathrm{H}^{+}$symporters (SUT/ SUC) enable the active transport of sucrose through membranes, driven by ATPase generated proton gradient (Carpaneto et al. 2005). SUTs are responsible for apoplastic phloem loading in source tissues and the active uptake of sugars into sink tissues including during grain filling [53]. Here we show that the transcript levels of both
ZmSWEET1 and ZmSUT1 tend to be induced by excision and in vitro culture, but also by 12 to $48 \mathrm{~h}$ of sucrose starvation. This indicate that these transporters could be involved in sugar uptake by the kernels, and that they might be regulated at the transcript level by sucrose availability. The slight elevation in ZmSWEET1 and ZmSUT1 transcripts under starvation might be a compensatory response of the kernel in order to import more sugars and enable survival. Evidence reviewed by Ayre [54] suggests that some sucrose transporters, such as ZmSUT1 [55], may be able to switch between functions of import and export depending on the needs of the specific plant tissue, allowing for separate functions in source and sink tissues. It has been suggested by Aoki et al. [56] that sucrose transporters may be required in non-photosynthetic organs to provide carbohydrates to growing tissues. Based on this assumption, the elevated mRNA observed in the starved kernels for ZmSUT1 and $Z m S W E E T 1$ may be tissue specific and important as reserves are consumed to provide for the metabolic needs of the plant experiencing sucrose starvation. Considering that this study separates the developing kernel from its physiological source tissue and that sucrose transporters are often tissue specific [56], it is not surprising that analysis on whole kernel samples will produce conflicting results as it measures all the components simultaneously.

\section{Role of Tre6P and the trehalose genes in the starvation response}

In context of the trehalose biosynthetic pathway, the results generally agree with what is known about this pathway. Tre6P levels have been shown to be highly correlated with sucrose (and Suc6P) concentration [18, 24, 57-60], and further shown to inhibit SnRK1 activity in developing tissues [20, 26, 27]. Yadav et al. [24] and Lunn et al. [18] proposed that, under a given set of conditions, the ratio of Tre6P to sucrose stays constant in that one controls the other in a Tre6P/sucrose nexus that regulates key metabolic processes important in the production and utilisation of sucrose and growth. Tre6P was shown to follow sucrose levels closer than any of the other sugars. They also proposed that that the sensitivity of the sucrose-Tre6P relationship is constant for a particular tissue, developmental stage and environmental condition, but can and does change if any of these three factors changes such as imposed stress [25, 57]. In the data presented here the correlation between sucrose and Tre6P did not always hold true. This likely resulted from a change in the condition or stage of the tissue between time zero and the $12 \mathrm{~h}$ time point. A similar interaction between sucrose and Tre6P levels has been observed before for shading stress in maize seedlings [61], and for salt stress in mature maize plants [39]. Based on Zhang et al. [20] who found that in vitro SnRK1 activity is 
inhibited by Tre6P at the concentrations found in kernels, it can be proposed that this large decrease of Tre6P will activate SnRK1 in both the control and starved kernels, presumably during the excision and preparation period $(2 \mathrm{~h})$. "The starved kernels later have a higher level of Tre6P-inhibitable SnRK1 activity, measured in vitro, indicating an additional mechanism may also promote SnRK1 activity in starved excised kernels. A similar interaction between sucrose and Tre6P levels has been observed before for shading stress in maize seedlings [61], and for salt stress in mature maize plants [39]. These findings add to the increasing evidence of the diverse functions of Tre6P and bring to light more of how little is known about this disaccharide and the need for further exploration of the trehalose pathway.

The results of this study agree mostly with the expected trends for the putative SnRK1 target genes. $\mathrm{Zm} \beta G$ al was an exception, being repressed initially; however, by $48 \mathrm{~h}$ there was no difference between the control and starved kernels. The correlation of Tre6P to SnRK1 target gene expression suggests that Tre6P inhibits SnRK1 activity. As the level of Tre6P drops during sucrose starvation there is a positive correlation with putative SnRK1 downregulated genes and a negative correlation with upregulated genes (Additional file 6: Table S3). The negative correlation between TPS class II genes and Tre6P is further evidence that Tre6P is involved in SnRK1 regulation as it is known that class II TPS genes are also targets of SnRK1 [27].

The responses of metabolites also support the activation of SnRK1 by starvation, and the involvement of the trehalose pathway in carbohydrate metabolism of kernels in the early stages of development. The reduction in sucrose synthesis, starch and citric acid cycle intermediates indicates a switch in metabolism consistent with an activation of SnRK1 as reviewed in [62-64]. The intermediary metabolite shikimate is an indicator of growth via its role in aromatic amino acid synthesis. During sucrose starvation shikimate levels are depleted. Low shikimate can be attributed as an indicator of growth arrest via the inhibition of key metabolic regulatory proteins (14-3-3 proteins) [65]. Glycolytic intermediates will be formed during sugar metabolism and also during catabolism, and their level will depend upon the relationship between the rate of formation and utilization. Interestingly, glycolytic intermediates were high in sucrose-fed kernels and in starved kernels, remained relatively high and rose further sucrose was re-supplied. This contrasts with organic acids and shikimate which remain low, indicating that the metabolic condition of the starved kernel continues in a state of growth arrest for the duration of the experiment. Perhaps if given more time for recovery the metabolites would return to control values. An alternative explanation is that the kernels have started down an irreversible path leading to abortion $[8,66]$ and will not recover.

The only intermediate that showed significantly increased levels during starvation was 3-PGA. 3-PGA levels were previously shown to decrease in maize leaves upon starvation induced by extended shading [57, 61]. When Arabidopsis seedlings were subjected to sucrose starvation and then fed sucrose in a recovery phase the seedlings experienced a decrease in 3-PGA during the first 3 days of recovery before levels increased [66]. Their study also showed rapid increases in sucrose, hexoses, sugar phosphates, and ATP. This observation is consistent with the recovery phase for kernels (Fig. 3). 3-PGA is important in both glycolysis and the Calvin cycle; however, we can presume that most of the 3PGA in the immature kernel comes from glycolysis. In glycolysis, 3-PGA is an important intermediate in the oxidation of hexose sugars to pyruvate to be used in either respiration or biosynthesis [67]. The build-up in PEP suggests that the consumption of this metabolite by pyruvate kinase and PEP carboxylase is restricted. Low PEP carboxylase activity could be due to the low Tre6P levels in excised kernels, given that high Tre6P leads to activation of this enzyme [68]. Via the reversible enolase and phosphoglycerate mutase reactions, accumulation of PEP would also be expected to lead to a similar accumulation of 3-PGA, accounting for the parallel increases in PEP and 3-PGA in excised kernels.

The build-up in 3-PGA may indicate the location of the restriction in glycolysis could be a result of lower phosphoglycerate mutase activity from repressed carbon availability. However, a more probable explanation is that the elevated 3-PGA concentration can be attributed to changes in glycerate availability from other sources in the plant tissue. The catabolic conversion of serine derived from the mitochondria and peroxisome may be the source of glycerate that would result in higher 3-PGA concentrations in sucrose deprived kernels [68].

\section{Role of the TPS gene family in the stress response}

In plants, the concentration of trehalose is too low to be useful as an osmoprotectant; however, the trehalose pathway has been shown to be essential in plants especially with regards to the activity of the TPS class I gene. The class I TPS (TPS1) has been confirmed to have enzymatic activity through complementation in yeast mutants deficient for TPS [69-71], and knocking out the AtTPS1 gene in Arabidopsis demonstrates its vital importance in embryo development and vegetative growth, and that the numerous class II TPSs are unable to compensate for the absence of TPS1 [72, 73]. In our experiments, the transcription of maize ZmTPSI.1.1 in 3 DAP kernels was found to be unresponsive to sucrose 
starvation. This is consistent with our previous findings that ZmTPSI.1.1 was unresponsive to salt stress in 3 DAP kernels [39]. A similar observation was made in Arabidopsis root and leaf tissue where AtTPS1 gene expression was found to be unresponsive to a wide range of abiotic stresses including drought, salt, cold, wounding, and heat [74]. This indicates that although it is essential to trehalose metabolism, TPS1 gene expression is not regulated at the transcript level. Given that the TPS1 gene is transcribed constitutively in most tissues, the catalytically active TPS is likely regulated via post-translational modification or binding of regulatory ligands $[75,76]$.

To date, the function of the class II TPS proteins remains unclear. As stated previously, they fail to reproducibly complement yeast TPS1 mutations, and they have thus far not been demonstrated to have in vitro enzymatic activity [71]. Regardless of the uncertainty of their function, class II TPSs show strict developmental and tissue specific gene regulation, strong diurnal regulation, and sensitivity to a variety of abiotic stressors $[39,74,77]$. The maize ZmTPSII.3.3 gene was shown to associate with the kernel expansion trait in popcorn [78]. The class II TPS proteins 5, 6 and 7 from Arabidopsis were found to be phosphoproteins and bind to 14-3-3 proteins when phosphorylated at Ser22 and Thr49 [76]. Unlike ZmTPSI.1.1, all of the maize class II TPS genes we followed were induced at the transcript level within the first $8 \mathrm{~h}$ of kernel culture, regardless of treatment. Class II TPS genes behaved similarly to the stress-inducible putative SnRK1 responsive genes with respect to the initial $2 \mathrm{~h}$ starvation, suggesting that SnRK1 has a role in the regulation of the class II genes in maize, as previously described in Arabidopsis [64].

\section{Tre6P Attenuates the Stress Response}

A two-hour period of starvation occurs for all samples between removal of the ear from the plant, though excising and surface sterilization of kernels, until plating on medium. Although tissue carbohydrate reserves are not completely depleted during this two hour imposed starvation stress, it is sufficient stimulus to see a significant impact on gene expression, particularly of putative SnRK1 target genes (Fig. 8). This effect on transcript levels is maintained throughout the experiment (96 h) for most genes regardless of whether or not sucrose is provided in the medium, likely because the Tre6P that typically attenuates the activity of SnRK1 and the expression of starvation responsive genes drops from a base level in the 3 DAP kernel of $54 \mathrm{nmol} \cdot \mathrm{g} \mathrm{FW}^{-1}$ to $<1 \mathrm{nmol} \cdot \mathrm{g} \mathrm{FW}{ }^{-1}$. Although there was a significant change in SnRK1 target gene expression in less than $12 \mathrm{~h}$ after kernels were excised from the cob, there was no significant change in total or Tre6P-inhibitable in vitro SnRK1 activity for control kernels. The activation of SnRK1 activity after kernel excision is likely a consequence of the precipitous drop in Tre6P levels, and not due to changes in SnRK1 protein levels. Actually total in vitro SnRK1 activity drops significantly after $12 \mathrm{~h}$ of starvation, even as SnRK1 is continuing to influence in vivo gene expression; although, in vitro Tre6P-inhibitable SnRK1 activity in sucrose starved kernels is significantly higher than in control kernels ( $>2$-fold). In many ways the imposed starvation stress in maize kernels parallels that which is seen in the maturing wheat kernel [58]. Tre6P levels drop precipitously, SnRK1 becomes derepressed, putative SnRK1 target genes are expressed, and significant changes in TPP and class II TPS gene expression are observed.
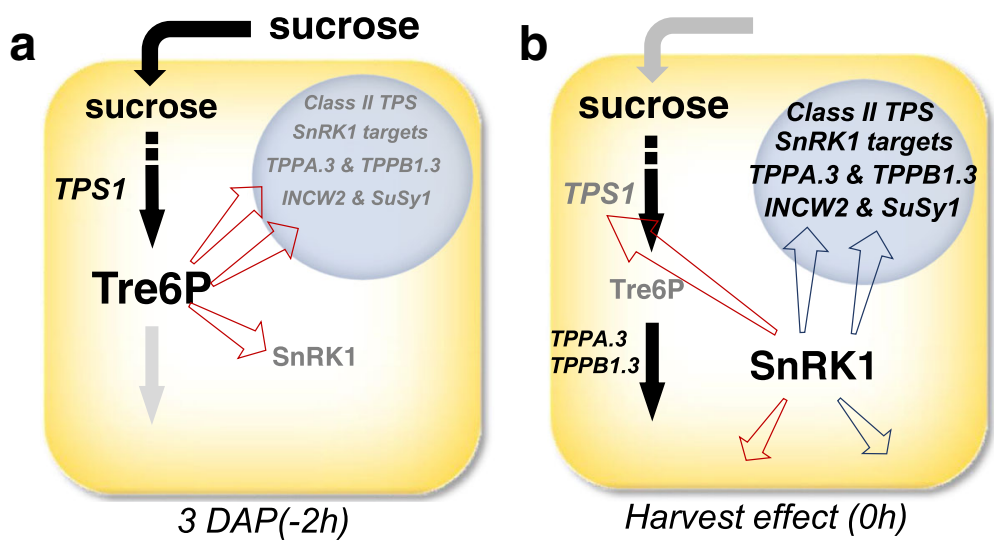

Fig. 8 This cartoon summarizes the events occurring during the two hours required to harvest, surface sterilize, and plate 3 DAP kernels on medium. In (a), kernels are on the plant being fed on sucrose obtained from photosynthesis. Cellular sucrose $\left(20 \mu \mathrm{mol} \cdot \mathrm{g}^{-1} \mathrm{FW}\right)$ and Tre6P levels are high (54 nmol.g ${ }^{-1} \mathrm{FW}$ ) and SnRK1 activity is low, as is the transcription of putative SnRK1 target genes, class II TPS genes, TPP genes, INCW2 and SuSy1. Two hours after ears are removed from the plant (b) Tre6P levels drop below $1 \mathrm{nmol} \cdot \mathrm{g}^{-1} \mathrm{FW}$, SnRK1 activity is high, and the transcription increases for putative SnRK1 target genes, class II TPS genes, TPP genes, INCW2 and SuSy1 
It is likely that the drop in Tre6P levels observed in starved maize kernels occurs because of the activation of one of the numerous trehalose phosphate phosphatases. All ten of the TPPs from Arabidopsis have been confirmed to have enzymatic activity through complementation of a yeast mutant [17]. Also, a chloroplastassociated redox-sensitive TPP from Arabidopsis (AtTPPD) was found to positively influence stress tolerance [79]. Salt had no effect on TPP gene expression in 3 DAP maize kernels [39]. The maize TPP RAMOSA 3 gene (ZmTPPB.2.1) is suspected to have a role in apical meristem growth and inflorescence development [80]. RAMOSA 3 is involved in branching in maize, and when RAMOSA 3 is knocked out of the maize genome unusual branching is often the result [81]. Each of the TPP genes responded differently to starvation stress, possibly indicating these genes have different functions in specific cell types found in a tissue sample [61]. It was observed; however, that starvation results in a large induction in the transcription of the ZmTPPA.3 gene. Recently, a rice ortholog of ZmTPPA.3 (OsTPP1) was ectopically over-expressed in maize pedicel tissue [33]. This resulted in a drop in ear spikelet (5 days before pollination) Tre6P levels from 48 to $18 \mathrm{nmol} \cdot \mathrm{g}$ $\mathrm{FW}^{-1}$, an increase in sucrose levels from 35 to $50 \mu \mathrm{mol} \cdot \mathrm{g}^{-1} \mathrm{FW}$, and improvement in drought tolerance. Interestingly, the protein coding sequence for ZmTPPA.3 was used to predict its subcellular localization (Wolf PSORT, Plant-mPLoc, and CropPlan), which suggested a high probability that the enzyme is localized to the chloroplast (data not shown). Tre6P is predominantly in the cytosol and a small amount associated with the chloroplast for Arabidopsis leaves grown in the light [82]. This suggests that Tre6P perhaps moves between subcellular compartments. This is consistent with a system of Tre6P transporters, similar to Glc6P transporters in E. coli $[83,84]$ and human [85]. It is currently not known if TPP is posttranslationally activated.

\section{Conclusions}

The impact of $48 \mathrm{~h}$ of starvation on 3 DAP maize kernels remains after two days' recovery on sucroseenriched medium. In the field this is equivalent to a rainfall being unable to reverse drought-induced kernel abortion. Future work will examine genetic variability amongst maize inbreds with respect to the capacity of kernels to recover from starvation.

Kernel excision triggers a 50-fold drop in kernel Tre6P, which does not recover even after $96 \mathrm{~h}$ on sucroseenriched medium. The removal of Tre6P suppression of SnRK1 activity results in the transcription of several putative SnRK1 target genes, and analyses of metabolite levels reveals a metabolic transition from biosynthesis to catabolism. This observation demonstrates the sensitivity of the maize inbred B73 to drought-induced kernel abortion, and highlights the importance of Tre6P in the metabolic response to starvation. We can speculate that the extremely high levels of Tre6P seen in the immature seed are needed to suppress SnRK1 activity, and further that the route to kernel abortion may begin with the precipitous drop in Tre6P and the resulting activation of SnRK1.

The drop in Tre6P corresponds to a large increase in transcription of ZmTPPA.3, indicating that this specific enzyme may be responsible for the de-phosphorylation of Tre6P, and thus a potential target for genetic and biotechnological improvement of drought tolerance in maize and other cereals.

\section{Methods}

\section{In vitro maize kernel culture}

This method was modified from the procedures of [34, 35, 37, 86] (Additional file 7: Figure S4). Metabolic and gene expression studies were performed in order to look at the impact of environmental conditions on kernel metabolism, verify that kernels continue to grow and develop normally in culture and determine the usefulness of this method as a surrogate for drought stress. In two sequential years the maize inbred B73 was grown in replicated plots with equal spacing and surrounded by a border to account for equal competition and shading. The maize inbred B73 was grown in replicated field plots in 2012 and 2013, watered twice a week to maintain available water content in the soil above 50\%. The growing seasons of 2012 and 2013 were noticeably different (Additional file 4: Table S2). The 2012 growing season was considered a drought year which saw the highest temperatures and growing degree days (GDD) compared for all three years. May and July in 2012 had the hottest days with average high temperatures 15 and $11 \%$ respectively above the mean, and GDDs 28 and $17 \%$ respectively above the 20-year mean. In addition, rainfall was the lowest for 2012 with precipitation 54\% lower than the mean. The 2013 growing season was milder with temperatures consistent within $4 \%$ and the GDD within $7 \%$ from the mean. Precipitation for 2013 was $16 \%$ below the mean. Each plot was replicated in three plantings, each a week apart to insure that enough pollen could be obtained. The plants were watered twice a week via sprinkler to minimize stress and ensure that available water content in the soil was kept above a 50\% threshold. Emerging shoots were covered by shoot bags to ensure no pollination can take place, and ears were pollinated by hand with B73 pollen. Three days after pollination, kernels were harvested from each of the 4 randomized rows from the most centrally located plants to ensure equal 
competition of available ground nutrients as well as shading effects. Ears were harvested, kept cool and hydrated in a beaker filled with water until preparation was complete $(<2 \mathrm{~h})$. Kernels were prepared by hand excision of the center one-third of the ear, and removal of kernels and a small portion of cob tissue. Sufficient cob tissue (cob to kernel ratio of 6:1) was required to permit nutrients to be taken up by the immature kernel [86]. The immature kernels were sterilized and grown in sucrose rich (150 mM sucrose, 1\% MS agar), or sucrose deficient media (1\% MS agar) on square petri dishes with equal spacing to ensure that there was no unequal competition between the developing kernels for the duration of the experiment [34, 35, 87]. The embryos were kept at $24{ }^{\circ} \mathrm{C}$ in the dark for $48 \mathrm{~h}$, then transferred to plates with sucrose for another $48 \mathrm{~h}$. Replicate samples are removed every 8-12 h, weighed and immediately frozen in liquid $\mathrm{N}_{2}$.

\section{Carbohydrate and metabolite analysis}

In order to evaluate the effect of sucrose starvation, excised kernels were cultured on MS medium without a carbon source for $48 \mathrm{~h}$ then allowed to recover on sucrose-enriched medium for another $48 \mathrm{~h}$. Kernels cultured for $96 \mathrm{~h}$ on continuous sucrose served as a control treatment. Frozen tissues $(20-100 \mathrm{mg})$ were ground to a fine powder (30-60s) with a Tissue Lyser II (Qiagen). Sucrose, fructose, and glucose were extracted using lactose as an internal standard [18]. Starch was extracted from the pellet generated during the extraction of soluble sugars, and hydrolyzed with amylase to produce glucose. All samples were analyzed by high-pressure capillary ion chromatograph system (ICS-5000, PA-20 column; ThermoFisher Dionex) using a $1 \mu$ l injection volume and $45 \mathrm{mM} \mathrm{KOH}$ eluent. Sugar peaks were identified in comparison with known sugars, and data were analyzed using the formula previously described with $\mathrm{L} / \mathrm{G}$ ratio of $1 / 100$ for kernel and cob [61]. T6P and other phosphorylated metabolites were quantified by anion-exchange liquid chromatography linked to tandem mass spectrometry [68].

\section{In vitro SnRK1 activity}

Kernel tissue $(200 \mathrm{mg})$ was ground in a mortar and pestle under liquid $\mathrm{N}_{2}$. Soluble proteins were extracted in $600 \mu \mathrm{L}$ of ice-cold homogenization buffer (100 mM Tricine- $\mathrm{NaOH}$, pH 8.0, $25 \mathrm{mM} \mathrm{NaF}, 5 \mathrm{mM}$ dithiothreitol, $2 \mathrm{mM}$ tetrasodium pyrophosphate, $0.5 \mathrm{mM}$ EDTA, $0.5 \mathrm{mM}$ EGTA, $1 \mathrm{mM}$ benzamidine, $1 \mathrm{mM}$ phenylmethylsulfonyl fluoride, $1 \mathrm{mM}$ protease inhibitor cocktail (Sigma P9599), phosphatase inhibitors (PhosStop; Roche) and insoluble polyvinylpyrrolidone to $2 \%(w / v)$. The homogenate was centrifuged $\left(13,000 \mathrm{~g}\right.$ at $\left.4{ }^{\circ} \mathrm{C}\right)$, and the supernatant $(250 \mu \mathrm{L})$ was desalted on Illustra NAP-5 columns (GE
Healthcare) pre-equilibrated with homogenization buffer. SnRK1 activity was determined as described [20]. Duplicate samples received Tre6P to a final concentration of $1 \mathrm{mM}$ to determine the percentage of in vitro SnRK1 activity inhibited by Tre6P.

\section{Messenger RNA analysis by qRT-PCR}

RNA isolation from maize tissue and qRT-PCR was performed as described in [61]. RT quality and absence of genomic DNA contamination was then checked by semi quantitative PCR on $5 \mu \mathrm{L}$ of cDNA (1/100 dilution) in a final volume of $25 \mu \mathrm{L}$, using ZmEF1-1 alpha primers (For: AGA CTC ACA TCA ACA TTG TGG TCA T, Rev.: GTT GTC ACC TTC AAA ACC AGA GAT T) designed around an intronic region and $\mathrm{GoTaq}^{\circ} \mathrm{DNA}$ Polymerase (Promega, USA) as recommended by the supplier. For each time point and biological replicate, qRT-PCR reaction was repeated 3 times. Experiments were performed on 3 biological replicates. Three out of 8 reference genes [39] were selected for normalization of gene expression using the Genorm software [88]. Relative gene expression was calculated using the method of [89]. Primer efficiency was determined using the method described by Pfaffl et al. [90].

\section{Additional files}

\begin{abstract}
Additional file 1: Figure S1. Kernels harvested 3 DAP were assessed for growth on various concentrations of sucrose, glucose and fructose during long term culture (15 d). After sterilization, kernels were plated on MS agar and incubated in the dark at $24^{\circ} \mathrm{C}$. medium contained either only MS salts, or MS salts supplemented with 100, 200 or 300 mM sucrose, glucose or fructose as a carbon source. Individual kernels were weighed at day zero of culture and at day 15. (XLSX $225 \mathrm{~kb}$ )
\end{abstract}

Additional file 2: Table S1. Kernels harvested 3 DAP were assessed for the effect of long term ( $9 \mathrm{~d}$ ) sucrose starvation on kernel growth $\left(\mathrm{g}^{-1} \mathrm{fw}\right)$ After sterilization, B73 kernels were plated on MS agar and incubated in the dark at $24{ }^{\circ} \mathrm{C}$. Control kernels on continuous sucrose are indicated by SUC (150 mM), and sucrose starved kernels are indicated by NS (no sucrose). Individual kernels from three seperate ears were weighed each day and returned to the medium. (XLSX $11 \mathrm{~kb}$ )

Additional file 3: Figure S2. Kernels harvested 3 DAP were tested for long term viability in culture. After sterilization, kernels were plated on MS agar and incubated in the dark at $24^{\circ} \mathrm{C}$. Control kernels on continuous sucrose $(150 \mathrm{mM})$ are indicated with closed (black) squares and sucrose starved kernels are indicated by white (open) squares. Means are plotted using 4 biological replicates consisting of 3 plants each. (XLSX 24 kb)

Additional file 4: Table S2. Growing conditions for field grown maize plants. Monthly and cumulative temperature $\left({ }^{\circ} \mathrm{C}\right)$, growing degree days (GDD) (base $10^{\circ} \mathrm{C}$ for Zea mays), and precipitation (cm) for the University of Nebraska-Lincoln campus field plots for the 2012, 2013, and 2014 growing seasons. The mean is determined on the 20-year average. (XLSX 15 kb)

Additional file 5: Figure S3. Effect of sucrose starvation on in vitro cultured kernels (3 DAP). Kernels were excised from field grown maize plants 3 days after pollination. After sterilization, kernels were plated on MS agar and incubated in the dark at $24^{\circ} \mathrm{C}$. Control kernels on continuous sucrose $(150 \mathrm{mM})$ are indicated with closed (black) squares and sucrose starved kernels are indicated by white (open) squares. The shaded area highlights the $48 \mathrm{~h}$ period of starvation, and the unshaded area indicates the period on recovery on sucrose. Means are calculated using 4 biological 
replicates consisting of 3 plants each, and significance as reported by the Student's T-test is at a $=0.05$. (XLSX $85 \mathrm{~kb}$ )

Additional file 6: Table S3. Heat map of correlation coefficients between Tre6P levels and gene expression. Coefficients of correlation were determined for control kernels continuously fed sucrose, and kernels starved for $48 \mathrm{~h}$ using a Pearson comparison test $(n-4)$. Positive correlations are indicated by shades of blue and negative correlations are indicated by shades of red. (XLSX $11 \mathrm{~kb}$ )

Additional file 7: Figure S4. Maize kernel culture. Kernels were prepared by hand excision of the center one-third of 3 DAP ears (A), and removal of kernels and a small portion of cob tissue (B), maintaining sufficient cob tissue to permit nutrients to be taken up by the immature kernel. Immature kernels were sterilized and grown in sucrose rich (150 mM Sucrose, 1\% MS agar), or sucrose deficient media (1\% MS agar) on petri dishes with equal spacing to ensure that there was equal competition for nutrients (C). Embryos were kept at $24{ }^{\circ} \mathrm{C}$ in the dark for $48 \mathrm{~h}$, then transferred to plates with sucrose for another $48 \mathrm{~h}$. Replicate samples are removed every $8-12 \mathrm{~h}$, kernels were removed from cob tissue (D \& E), weighed, and immediately frozen in liquid $N_{2}$. (XLSX $310 \mathrm{~kb}$ )

\section{Abbreviations}

3-PGA: 3-phosphoglyceraldehyde; ADP-Glc: Adenine diphosphate glucoside; ASI: Anthesis silking interval; DAA: Days after anthesis; DAP: Days after pollination; FBP: Fructose bisphosphate; Fru: Fructose; Fru6P: Fructose-6phosphate; GDD: Growing degree days; Glc: Glucose; Glc1P: Glucose-1phosphate; Glc6P: Glucose-6-phosphate; Gly3P: Glyceraldehyde-3-phosphate; HPIC: High pressure ion chromatography; HPIC: High pressure ion chromatography; SnRK1: Sucrose non-fermenting-1-Related protein Kinase 1; TPP: Trehalose phosphate phosphatase; TPS: Trehalose phosphate synthase; TRE: Trehalase; Tre6P: Trehalose-6-phosphate; UDPG: Uridine diphosphate glucose

\section{Acknowledgements}

None.

\section{Funding}

Not applicable.

\section{Availability of data and materials}

Additional data are available in Additional files 1, 2, 3, 4, 5, 6 and 7).

\section{Authors' contributions}

SWB performed kernel culture, HPIC analysis of sugars, RT-PCR, and the data analysis. CAG performed the in vitro SnRK1 assay. RF performed the HPICMS-MS analysis of sugar phosphates. CH, MJP, MS and JL assisted in writing and editing the manuscript. SWB and LML designed the experiments and wrote the paper.

\section{Competing interests}

The authors declare that they have no competing interests.

\section{Consent for publication}

Not applicable.

\section{Ethics approval and consent to participate}

Not applicable.

\section{Publisher's Note}

Springer Nature remains neutral with regard to jurisdictional claims in published maps and institutional affiliations.

\section{Author details}

'EAG Laboratories, 4780 Discovery Drive, Columbia, MO 65201, USA. ${ }^{2}$ Hawkesbury Institute for the Environment, Western Sydney University, Locked Bag 1797, Penrith, NSW 2751, Australia. ${ }^{3}$ Rothamsted Research, West Common, Harpenden, Hertfordshire AL5 2JQ, UK. ${ }^{4}$ Max Planck Institut für Moleckulare Pflanzenphysiologie, Potsdam (OT) Golm, Germany. ${ }^{5}$ Department of Agronomy and Horticulture, University of Nebraska-Lincoln, 377I Plant Science, Lincoln, NE 68583-0915, USA.
Received: 5 December 2016 Accepted: 24 March 2017

Published online: 12 April 2017

\section{References}

1. Westgate ME, Boyer JS. Carbohydrate Reserves and Reproductive Development at Low Leaf Water Potentials in Maize1. Crop Sci. 1985;25:762.

2. Westgate ME, Boyer JS. Reproduction at Low and Pollen Water Potentials in Maize1. Crop Sci. 1986;26:951

3. Otegui ME, Andrade FH, Suero EE. Growth, water use, and kernel abortion of maize subjected to drought at silking. Field Crop Res. 1995;40:87-94.

4. Khorasani S, Mostafavi K, Heidarian A. Response of Maize (Zea mays L.) hybrids and inbred lines to salinity stress under field condition. Tech J Eng Appl Sci. 2011:2:1-7.

5. Hiyane R, Hiyane S, Tang AC, Boyer JS. Sucrose feeding reverses shadeinduced kernel losses in maize. Ann Bot. 2010;106:395-403.

6. McLaughlin JE, Boyer JS. Glucose localization in maize ovaries when kerne number decreases at low water potential and sucrose is fed to the stems. Ann Bot. 2004:94:75-86.

7. Boyle MG, Boyer JS, Morgan PW. Stem Infusion of Liquid Culture Medium Prevents Reproductive Failure of Maize at Low Water Potential. Crop Sci. 1991;31:1246-57.

8. McLaughlin JE, Boyer JS. Sugar-responsive Gene Expression, Invertase Activity, and Senescence in Aborting Maize Ovaries at Low Water Potentials. Ann Bot. 2004:94:675-89.

9. Elbein AD, Pan YT, Pastuszak I, Carroll D. New insights on trehalose: a multifunctional molecule. Glycobiology. 2003;13:17R-27R

10. Crowe JH, Hoekstra FA, Crowe LM. Anhydrobiosis. Annu Rev Physiol. 1992; 54:579-99.

11. Birch GG. TREHALOSES. Adv Carbohydr Chem. 1963;18:201.

12. Crowe JH. Trehalose as a "chemical chaperone": fact and fantasy. Adv Exp Med Biol. 2007:594:143-58.

13. Brodmann A, Schuller A, Ludwig-Müller J, Aeschbacher RA, Wiemken A, Boller T, Wingler A. Induction of trehalase in Arabidopsis plants infected with the trehalose-producing pathogen Plasmodiophora brassicae. Mol Plant-Microbe Interact. 2002:15:693-700

14. Avonce N, Mendoza-Vargas A, Morett E, Iturriaga G. Insights on the evolution of trehalose biosynthesis. BMC Evol Biol. 2006:6:109-24.

15. Paul MJ, Primavesi LF, Jhurreea D, Zhang Y. Trehalose metabolism and signaling. Annu Rev Plant Biol. 2008:59:417-41.

16. Gómez LD, Baud S, Gilday A, Li Y, Graham IA. Delayed embryo development in the ARABIDOPSIS TREHALOSE-6-PHOSPHATE SYNTHASE 1 mutant is associated with altered cell wall structure, decreased cell division and starch accumulation. Plant J. 2006:46:69-84

17. Vandesteene L, López-Galvis L, Vanneste K, Feil R, Maere S, Lammens W, Rolland F, Lunn JE, Avonce N, Beeckman T, Van Dijck P. Expansive evolution of the trehalose-6-phosphate phosphatase gene family in arabidopsis. Plant Physiol. 2012;160:884-96.

18. Lunn JE, Feil R, Hendriks JHM, Gibon Y, Morcuende R, Osuna D, Scheible W$R$, Carillo P, Hajirezaei M-R, Stitt M. Sugar-induced increases in trehalose 6phosphate are correlated with redox activation of ADPglucose pyrophosphorylase and higher rates of starch synthesis in Arabidopsis thaliana. Biochem J. 2006:397:139-48.

19. Paul M. Trehalose 6-phosphate. Curr Opin Plant Biol. 2007;10:303-9.

20. Zhang Y, Primavesi LF, Jhurreea D, Andralojc PJ, Mitchell RAC, Powers SJ, Schluepmann H, Delatte T, Wingler A, Paul MJ. Inhibition of SNF1-related protein kinase 1 activity and regulation of metabolic pathways by trehalose6-phosphate. Plant Physiol. 2009;149:1860-71.

21. Ponnu J, Wahl V, Schmid M. Trehalose-6-phosphate: connecting plant metabolism and development. Front Plant Sci. 2011;2:70.

22. Wingler A, Delatte $T L, O^{\prime}$ Hara LE, Primavesi LF, Jhurreea D, Paul MJ, Schluepmann $\mathrm{H}$. Trehalose 6-phosphate is required for the onset of leaf senescence associated with high carbon availability. Plant Physiol. 2012 158:1241-51.

23. Wahl V, Ponnu J, Schlereth A, Arrivault S, Langenecker T, Franke A, Feil R, unn JE, Stitt M, Schmid M. Regulation of flowering by trehalose-6phosphate signaling in Arabidopsis thaliana. Science. 2013;339:704-7.

24. Yadav UP, Ivakov A, Feil R, Duan GY, Walther D, Giavalisco P, Piques M, Carillo P, Hubberten H-M, Stitt M, Lunn JE. The sucrose-trehalose 6phosphate (Tre6P) nexus: specificity and mechanisms of sucrose signalling by Tre6P. J Exp Bot. 2014;65:1051-68. 
25. Figueroa CM, Lunn JE. A tale of two sugars - trehalose 6-phosphate and sucrose. Plant Physiol. 2016;172(September):7-27.

26. Paul MJ, Jhurreea D, Zhang Y, Primavesi LF, Delatte T, Schluepmann H, Wingler A. Upregulation of biosynthetic processes associated with growth by trehalose 6-phosphate. Plant Signal Behav. 2010;5:386-92.

27. Baena-González E, Rolland F, Thevelein JM, Sheen J. A central integrator of transcription networks in plant stress and energy signalling. Nature. 2007; 448:938-42.

28. Wingler A. The function of trehalose biosynthesis in plants. Planta. 2002;60:437-40.

29. Lunn JE, Delorge I, Figueroa CM, Van Dijck P, Stitt M. Trehalose metabolism in plants. Plant J. 2014:49:544-67.

30. Holmström K, Mäntylä E, Welin B, Mandal A. Drought tolerance in tobacco. Nature. 1996:379:683-4.

31. Garg AK, Kim J-K, Owens TG, Ranwala AP, Choi YD, Kochian LV, Wu RJ. Trehalose accumulation in rice plants confers high tolerance levels to different abiotic stresses. Proc Natl Acad Sci. 2002;99:15898-903.

32. Karim S, Aronsson $H$, Ericson $H$, Pirhonen M, Leyman B, Welin B, Mäntylä $E_{\text {, }}$ Palva ET, Van Dijck P, Holmström K-O. Improved drought tolerance without undesired side effects in transgenic plants producing trehalose. Plant Mol Biol. 2007;64:371-86.

33. Nuccio ML, Wu J, Mowers R, Zhou H-P, Meghii M, Primavesi LF, Paul MJ, Chen X, Gao Y, Haque E, Basu SS, Lagrimini LM. Expression of trehalose-6phosphate phosphatase in maize ears improves yield in well-watered and drought conditions. Nat Biotechnol 2015;(October 2014):1-13.

34. Hanft JM, Jones RJ. Kernel abortion in maize : I. Carbohydrate concentration patterns and Acid invertase activity of maize kernels induced to abort in vitro. Plant Physiol. 1986;81:503-10.

35. Glawischnig E, Tomas A, Eisenreich W, Spiteller P, Bacher A, Gierl A. Auxin biosynthesis in maize kernels. Plant Physiol. 2000;123:1109-19.

36. Hole DJ, Smith JD, Cobb BG. Regulation of Embryo Dormancy by Manipulation of Abscisic Acid in Kernels and Associated Cob Tissue of Zea mays L. Cultured in Vitro. Plant Physiol. 1989;91:101-5.

37. Singletary GW, Below FE. Growth and Composition of Maize Kernels Cultured in Vitro with Varying Supplies of Carbon and Nitrogen. Plant Physiol. 1989;89:341-6.

38. Gengenbach BG. Development of maize caryopses resulting from in-vitro pollination. Planta. 1977;134:91-3.

39. Henry C, Bledsoe SW, Griffiths CA, Kollman A, Paul MJ, Sakr S, Lagrimini LM. Differential Role for Trehalose Metabolism in Salt-Stressed Maize. Plant Physiol. 2015;169:1072-89.

40. Duke ER, Doehlert DC. Effects of heat stress on enzyme activities and transcript levels in developing maize kernels grown in culture. Environ Exp Bot. 1996:36:199-208.

41. Zinselmeier C, Lauer M, Boyer JS. Reversing Drought-Induced Losses in Grain Yield: Sucrose Maintains Embryo Growth in Maize. Crop Sci. 1995;35: 1390-9.

42. Koch K. Sucrose metabolism: regulatory mechanisms and pivotal roles in sugar sensing and plant development. Curr Opin Plant Biol. 2004;7:235-46.

43. Jones RJ, Setter TL. Hormonal regulation of early kernel development. In: M. Westgate, K. Boote, editors, Physiology and Modeling Kernel Set in Maize, CSSA Spec. Publ. 29. CSSA and ASA, Madison. 2000. pp. 25-42. doi:10.2135/ cssaspecpub29.c3.

44. Stark DM, Timmerman KP, Barry GF, Preiss J, Kishore GM. Regulation of the Amount of Starch in Plant Tissues by ADP Glucose Pyrophosphorylase. Science. 1992;258:287-92.

45. Ekkehard Neuhaus H, Stitt M. Control analysis of photosynthate partitioning: Impact of reduced activity of ADP-glucose pyrophosphorylase or plastid phosphoglucomutase on the fluxes to starch and sucrose Inarabidopsis thaliana (L.) Heynh. Planta. 1990;182:445-54.

46. Preiss J. Regulation of the biosynthesis and degradation of starch. Plant Physiol. 1982:33:431-54.

47. Zeeman SC, Smith S, Smith A. The diurnal metabolism of leaf starch. Biochem J. 2007:401:13-28

48. Andersen $M$, Asch $F$, Wu Y. Soluble invertase expression is an early target of drought stress during the critical, abortion-sensitive phase of young ovary development in maize. Plant Physiol. 2002;130:591-604

49. Zinselmeier C. Starch and the Control of Kernel Number in Maize at Low Water Potentials. Plant Physiol. 1999;121:25-36.

50. Zinselmeier C, Westgate ME, Schussler JR, Jones RJ. Low Water Potential Disrupts Carbohydrate Metabolism in Maize (Zea mays L.) Ovaries. Plant Physiol. 1995;107:385-91.
51. Chen L-Q. SWEET sugar transporters for phloem transport and pathogen nutrition. New Phytol. 2014;201:1150-5.

52. Chen L-Q, Qu X-Q, Hou B-H, Sosso D, Osorio S, Fernie AR, Frommer WB. Sucrose efflux mediated by SWEET proteins as a key step for phloem transport. Science. 2012;335:207-11.

53. Slewinski TL, Garg A, Johal GS, Braun DM. Maize SUT1 functions in phloem loading. Plant Signal Behav. 2010;5(June):687-90.

54. Ayre BG. Membrane-transport systems for sucrose in relation to whole-plant carbon partitioning. Mol Plant. 2011;4:377-94.

55. Carpaneto A, Geiger D, Bamberg E, Sauer N, Fromm J, Hedrich R. Phloemlocalized, proton-coupled sucrose carrier ZmSUT1 mediates sucrose efflux under the control of the sucrose gradient and the proton motive force. J Biol Chem. 2005;280:21437-43.

56. Aoki N, Hirose T, Takahashi S, Ono K, Ishimaru K, Ohsugi R. Molecular Cloning and Expression Analysis of a Gene for a Sucrose Transporter in Maize (Zea mays L.). Plant Cell Physiol. 1999;40:1072-8.

57. Czedik-Eysenberg AB, Arrivault S, Lohse MA, Feil R, Krohn N, Encke B, NunesNesi A, Fernie AR, Lunn JE, Sulpice R, Stitt M. The interplay between carbon availability and growth in different zones of the growing maize leaf. Plant Physiol. 2016;172:943-67.

58. Martínez-Barajas E, Delatte T, Schluepmann H, de Jong GJ, Somsen GW Nunes C, Primavesi LF, Coello P, Mitchell RA, Paul MJ. Wheat grain development is characterized by remarkable trehalose 6-phosphate accumulation pregrain filling: tissue distribution and relationship to SNF1related protein kinase1 activity. Plant Physiol. 2011;156:373-81.

59. Schluepmann $H$, van Dijken A, Aghdasi M, Wobbes B, Paul MJ, Smeekens SC. Trehalose mediated growth inhibition of Arabidopsis seedlings is due to trehalose-6-phosphate accumulation. Plant Physio. 2004;135:879-90.

60. Delatte TL, Sedijani P, Kondou Y, Matsui M, de Jong GJ, Somsen GW, WieseKlinkenberg A, Primavesi LF, Paul MJ, Schluepmann H. Growth Arrest by Trehalose-6-Phosphate: An Astonishing Case of Primary Metabolite Control over Growth by Way of the SnRK1 Signaling Pathway. Plant Physiol. 2011;157:160-74.

61. Henry C, Bledsoe SW, Siekman A, Kollman A, Waters BM, Feil R, Stitt M, Lagrimini LM. The trehalose pathway in maize: conservation and gene regulation in response to the diurnal cycle and extended darkness. J Exp Bot. 2014;65:5959-73.

62. Schluepmann H, Berke L, Sanchez-Perez GF. Metabolism control over growth: a case for trehalose-6-phosphate in plants. J Exp Bot. 2011;63:3379-90.

63. Delatte TL, Selman MHJ, Schluepmann H, Somsen GW, Smeekens SCM, de Jong GJ. Determination of trehalose-6-phosphate in Arabidopsis seedlings by successive extractions followed by anion exchange chromatographymass spectrometry. Anal Biochem. 2009;389:12-7.

64. Baena-González E. Energy signaling in the regulation of gene expression during stress. Mol Plant. 2010;3:300-13.

65. Diaz C, Kusano M, Sulpice R, Araki M, Redestig H, Saito K, Stitt M, Shin R. Determining novel functions of Arabidopsis 14-3-3 proteins in central metabolic processes. BMC Syst Biol. 2011:5:192.

66. Lauxmann MA, Annunziata MG, Brunoud G, Wahl V, Koczut A, Burgos A, Olas JJ, Maximova E, Abel C, Schlereth A, Soja AM, Bläsing OE, Lunn JE, Vernoux T, Stitt M. Reproductive failure in Arabidopsis thaliana under transient carbohydrate limitation: Flowers and very young siliques are jettisoned and the meristem is maintained to allow successful resumption of reproductive growth. Plant Cell Environ. 2016;39:745-67.

67. Plaxton WC. THE ORGANIZATION AND REGULATION OF PLANT GLYCOLYSIS. Annu Rev Plant Physiol Plant Mol Biol. 1996;47:185-214.

68. Figueroa CM, Feil R, Ishihara I, Watanabe M, Kölling K, Krause U, Höhne M, Encke B, Plaxton WC, Zeeman SC, Li Z, Schulze WX, Hoefgen R, Stitt M, Lunn JE. Trehalose 6 - phosphate coordinates organic and amino acid metabolism with carbon availability. Plant J. 2016;85:410-23.

69. Jiang W, Fu F-L, Zhang S-Z, Wu L, Li W-C. Cloning and Characterization of Functional Trehalose-6-Phosphate Synthase Gene in Maize. J Plant Biol. 2010;53:134-41.

70. Vogel G, Aeschbacher RA, Müller J, Boller T, Wiemken A. Trehalose-6-phosphate phosphatases from Arabidopsis thaliana: identification by functional complementation of the yeast tps2 mutant. Plant J Cell Mol Biol. 1998;13:673-83.

71. Zang B, Li H, Li W, Deng XW, Wang X. Analysis of trehalose-6-phosphate synthase (TPS) gene family suggests the formation of TPS complexes in rice. Plant Mol Biol. 2011;76:507-22.

72. van Dijken AJH, Schluepmann H, Smeekens SCM. Arabidopsis trehalose-6phosphate synthase 1 is essential for normal vegetative growth and transition to flowering. Plant Physiol. 2004;135:969-77. 
73. Gómez LD, Gilday A, Feil R, Lunn JE, Graham IA. AtTPS1-mediated trehalose 6-phosphate synthesis is essential for embryogenic and vegetative growth and responsiveness to $A B A$ in germinating seeds and stomatal guard cells. Plant J. 2010:64:1-13.

74. Iordachescu M, Imai R. Trehalose biosynthesis in response to abiotic stresses. J Integr Plant Biol. 2008:50:1223-9.

75. Glinski M, Weckwerth W. Differential multisite phosphorylation of the trehalose-6-phosphate synthase gene family in Arabidopsis thaliana: a mass spectrometry-based process for multiparallel peptide library phosphorylation analysis. Mol Cell Proteomics. 2005;4:1614-25.

76. Harthill JE, Meek SEM, Morrice N, Peggie MW, Borch J, Wong BHC, Mackintosh C. Phosphorylation and 14-3-3 binding of Arabidopsis trehalose-phosphate synthase 5 in response to 2-deoxyglucose. Plant J. 2006;47:211-23.

77. Ramon M, De Smet I, Vandesteene L, Naudts M, Leyman B, Van Dijck P, Rolland F, Beeckman T, Thevelein JM. Extensive expression regulation and lack of heterologous enzymatic activity of the Class II trehalose metabolism proteins from Arabidopsis thaliana. Plant Cell Environ. 2009;32:1015-32.

78. Paes GP, Viana JMS, Silva FFE, Mundim GB. Linkage disequilibrium, SNP frequency change due to selection, and association mapping in popcorn chromosome regions containing QTLs for quality traits. Genet Mol Biol. 2016;39:97-110.

79. Krasensky J, Broyart C, Rabanal FA, Jonak C. The Redox-Sensitive Chloroplast Trehalose-6-Phosphate Phosphatase AtTPPD Regulates Salt Stress Tolerance. Antioxid Redox Signal. 2014;21:1-16.

80. Satoh-Nagasawa N, Nagasawa N, Malcomber S, Sakai H, Jackson D. A trehalose metabolic enzyme controls inflorescence architecture in maize. Nature. 2006;441:227-30.

81. McSteen P. Branching out: the ramosa pathway and the evolution of grass inflorescence morphology. Plant Cell. 2006;18:518-22.

82. Martins MCM, Hejazi M, Fettke J, Steup M, Feil R, Krause U, Arrivault S, Vosloh D, Figueroa CM, Ivakov A, Yadav UP, Piques M, Metzner D, Stitt M, Lunn JE. Feedback inhibition of starch degradation in Arabidopsis leaves mediated by trehalose 6-phosphate. Plant Physiol. 2013;163:1142-63.

83. Lloyd AD, Kadner RJ. Topology of the Escherichia coli uhpT sugar-phosphate transporter analyzed by using TnphoA fusions. J Bacteriol. 1990;172:1688-93.

84. Weston LA, Kadner RJ. Role of uhp genes in expression of the Escherichia coli sugar-phosphate transport system. J Bacteriol. 1988;170:3375-83.

85. Chou JY, Mansfield BC. The SLC37 Family of Sugar-Phosphate/phosphate Exchangers. 1st edition. Volume 73. Elsevier Inc.; 2014.

86. Felker FC. Participation of Cob Tissue in the Uptake of Medium Components by Maize Kernels Cultured In Vitro. J Plant Physiol. 1992;139: 647-52.

87. Muhitch MJ, Shatters RG. Regulation of the maize ubiquitin (Ubi-1) promoter in developing maize ( Zea mays L.) seeds examined using transient gene expression in kernels grown in vitro. Plant Cell Rep. 1998;17:476-81.

88. Vandesompele J, De Preter K, Pattyn F, Poppe B, Van Roy N, De Paepe A Speleman F. Accurate normalization of real-time quantitative RT-PCR data by geometric averaging of multiple internal control genes. Genome Biol. 2002;3:research0034.1-research0034.11.

89. Hellemans J, Mortier G, De Paepe A, Speleman F, Vandesompele J. aBase relative quantification framework and software for management and automated analysis of real-time quantitative PCR data. Genome Biol. 2007;8:R19.

90. Pfaffl MW. A new mathematical model for relative quantification in real-time RT-PCR. Nucleic Acids Res. 2001;29:e45.

\section{Submit your next manuscript to BioMed Central and we will help you at every step:}

- We accept pre-submission inquiries

- Our selector tool helps you to find the most relevant journal

- We provide round the clock customer support

- Convenient online submission

- Thorough peer review

- Inclusion in PubMed and all major indexing services

- Maximum visibility for your research

Submit your manuscript at www.biomedcentral.com/submit 